\title{
Irisin reverses the IL-6 induced epithelial-mesenchymal transition in osteosarcoma cell migration and invasion through the STAT3/Snail signaling pathway
}

\author{
GANG KONG $^{1 *}$, YUNPENG JIANG $^{2 *}$, XIUJIANG SUN ${ }^{1}$, ZHILIN CAO $^{1}$, GUODONG ZHANG $^{1}$, \\ ZHONGYUAN ZHAO ${ }^{1}$, YONG ZHAO ${ }^{1}$, QIAN YU $^{1}$ and GONG CHENG $^{1}$ \\ ${ }^{1}$ Department of Orthopaedics, Yantaishan Hospital, Yantai, Shandong 264001; \\ ${ }^{2}$ Department of Orthopaedics, Qilu Hospital of Shandong University, Jinan, Shandong 250000, P.R. China
}

Received March 8, 2017; Accepted August 9, 2017

DOI: $10.3892 /$ or.2017.5973

\begin{abstract}
As a novel discovered myokine, irisin is considered to be a promising candidate for the treatment of metabolic disorders and cancer. However, little is known about the anti-metastasic effect of irisin on osteosarcoma cells and its underlying mechanisms. In the present study, we aimed to explore the effect of irisin on the migration and invasion of osteosarcoma cells and the underlying mechanisms involved. Viability and proliferation of osteosarcoma cells were examined by MTT assay. Then, by using scratch wound healing assay and Transwell assays, we evaluated migratory and invasive ability of the cells, respectively. Moreover, the expression of epithelial-to-mesenchymal transition (EMT) markers were determined by qPCR, western blot and immunofluorescence staining after treatment with IL- 6 and irisin. Furthermore, the expression of ERK, p38, STAT3 and Snail were detected by western blot analysis. Finally, an inhibitor of STAT3, WP1066 was applied to testify the effect of irisin on the expression of EMT markers and Snail. It was found that irisin treatment significantly suppressed the proliferation, migration and invasion of osteosarcoma cells. Furthermore, irisin reversed the IL-6-induced epithelial-mesenchymal transition (EMT) in osteosarcoma cells by regulating the expression of E-cadherin, $\mathrm{N}$-cadherin, vimentin, fibronectin, MMP-2, MMP-7 and MMP-9. In addition, irisin suppressed the IL-6-activated phosphorylation of STAT3 and the expression of Snail in osteosarcoma cells. Finally, blockade of STAT3 by WP1066 (a STAT3 inhibitor) further enhanced the effect of irisin on the
\end{abstract}

Correspondence to: Dr Gong Cheng, Department of Orthopaedics, Yantaishan Hospital, 91 Jiefang Road, Yantai, Shandong 264001, P.R. China

E-mail: chenggongsubmit@qq.com

${ }^{*}$ Contributed equally

Key words: irisin, epithelial-mesenchymal transition, osteosarcoma cells, STAT3, Snail
EMT and Snail expression in osteosarcoma cells. Collectively, our findings revealed that irisin may play a critical role in the IL-6-induced EMT of osteosarcoma cells via the STAT3/Snail signaling pathway.

\section{Introduction}

It has been well documented that exercise has many potential benefits in chronic metabolic diseases including type II diabetes mellitus and metabolic syndrome, cardiovascular disease and cancer (1-4). Recently, studies have focused on the benefits of exercise in various types of cancers, such as breast, lung and gynaecological cancer (5-7). However the potential underlying mechanisms of exercise on cancer treatment and improved quality of life are not well established.

In recent years, skeletal muscle is gaining increased attention as an endocrine organ (8). Various myokines which regulate beneficial effects have been identified, such as irisin, a newly discovered myokine, which is secreted from the skeletal muscles following exercise (9). Irisin is believed to be a bridge that links exercise with increased energy expenditure. Studies have documented that irisin is released upon cleavage of the membrane of fibronectin type III domain-containing protein 5 (FNDC5) and is increased with exercise $(9,10)$. Previous studies have demonstrated the role of irisin in body energy expenditure and in insulin sensitivity $(9,10)$. Furthermore, irisin has been demonstrated to play a role in the mediation of H19-7 hippocampal neuronal cell proliferation (11) and in the differentiation of adipocytes and osteoblasts (12-14). In addition to these studies, irisin has been demonstrated to have a protective role in vascular pathology by regulating endothelial cell proliferation, apoptosis and migration, as well as smooth muscle cell phenotype modulation (15-18). Notably, recent studies have focused on the relationship between irisin and cancer. A recent study found that serum irisin levels were lower in patients with breast cancer (19), while other studies revealed that irisin was significantly increased in gastrointestinal cancer tissues $(20,21)$. In addition, studies have revealed increased irisin immunoreactivity in tissues which were obtained from ovary, cervix and breast carcinomas and endometrial hyperplasias (22). 
Moreover, a recent study demonstrated that irisin significantly inhibited the viability and migration and enhanced the tumor sensitivity to doxorubicin (DOX) in malignant breast cancer cells (23). Additionally another study revealed the suppressive effect of irisin in lung cancer cell migration and invasion (24). These studies underlined the critical role of irisin in carcinogenesis. Furthermore, these findings may offer therapeutic benefits for cancer prevention and treatment.

At present, osteosarcoma, the most common primary bone malignant tumor, has a poor prognosis due to distal metastases (25). Although chemotherapy is combined with surgery to improve the prognosis, the survival rate is still low (26). Therefore it is urgent to identify novel drugs for a better treatment outcome in osteosarcoma therapy.

Osteosarcoma cells possess highly invasive properties by undergoing a unique phenotypic switch, epithelial-mesenchymal transition (EMT) (27). EMT is thought to be a highly conserved cellular process which is characterized by inhibition of epithelial molecule E-cadherin and gaining of mesenchymal markers, such as $\mathrm{N}$-cadherin, vimentin and fibronectin (28). Studies have revealed the critical role of EMT in carcinoma metastasis $(28,29)$.

It has been well established that IL-6 promotes the proliferation, metastasis and angiogenesis of osteosarcoma $(30,31)$. Studies further demonstrated that IL-6 induced EMT in various types of cancer cells, such as pancreatic (32), lung (33), hepatocellular (34) and colorectal cancer cells (35). The IL-6 downstream signals included STAT3, Akt and ERK1/2 MAPK (30,36,37). Among them, STAT3 has been demonstrated to exhibit an important role in IL-6-modulated EMT $(33,38)$. Previous studies have revealed the critical role of STAT3 in modulating angiogenesis and metastasis of cancer $(39,40)$. Furthermore, studies have revealed that the Snail family members, including Snail, ZEB1, Twist, Slug and SIP1, played a crucial role in regulating EMT (41).

Thus, the present study evaluated the effect of irisin on osteosarcoma cell migration, invasion and EMT and explored the mechanisms involved.

\section{Materials and methods}

Cell lines and reagents. The U2OS and MG-63 osteosarcoma cell lines were purchased from the American Type Culture Collection (ATCC, Manassas, VA, USA) and were routinely cultured in Dulbecco's modified Eagle's medium (DMEM; Invitrogen Life Technologies, Carlsbad, CA, USA) containing 10\% FBS (Invitrogen Life Technologies), 100 U/ml penicillin, and $100 \mu \mathrm{g} / \mathrm{ml}$ streptomycin in $5 \% \mathrm{CO}_{2}$ at $37^{\circ} \mathrm{C}$. Rabbit anti-STAT3, phosphor-STAT3, rabbit anti-ERK1/2, phosphor-ERK1/2, rabbit anti-p38, phosphor-p38, rabbit anti-MMP-2, rabbit anti-MMP-9 and rabbit anti-Snail antibodies were all purchased from Cell Signaling Technology (Danvers, MA, USA). Rabbit anti-E-cadherin, vimentin, $\mathrm{N}$-cadherin, fibronectin and rabbit anti-MMP-7 antibodies were purchased from Abcam (Cambridge, UK). Alexa Fluor 488-conjugated goat anti-rabbit IgG was purchased from Invitrogen Life Technologies. IL-6 was purchased from PeproTech (Rocky Hill, NJ, USA). Irisin was purchased from Phoenix Pharmaceuticals (Burlingame, CA, USA). WP1066, a selective inhibitor of STAT3, was purchased from Sigma-Aldrich (St. Louis, MO, USA).
Cell viability assay. 3-(4,5-Dimethylthiazol-2-yl)-2,5diphenyltetrazolium bromide (MTT) assay was used to assess the cell proliferation and viability. The U2OS and MG-63 osteosarcoma cells (5,000 cells/well) in $100 \mu \mathrm{l}$ medium were seeded into 96 -well plates. After being stimulated with irisin at different doses $(0,25,50,100$ and $200 \mathrm{ng} / \mathrm{ml})$ for different time-points (12, 24 and $48 \mathrm{~h}), 20 \mu \mathrm{l}$ MTT solution $(5 \mathrm{mg} / \mathrm{ml})$ was added into each well. After incubation for $4 \mathrm{~h}, 100 \mu \mathrm{l}$ of dimethyl sulfoxide (DMSO) was added to each well for another $15 \mathrm{~min}$. Then, the absorbance values were determined using a microplate reader (Thermo Fisher Scientific, Inc., Waltham, MA, USA) at $490 \mathrm{~nm}$.

Scratch wound healing assay. To evaluate the migration of the U2OS and MG-63 osteosarcoma cells, a scratch wound healing assay was used. Briefly, the U2OS and MG-63 cells (1x10\%/well) were seeded in 6-well plates and cultured with DMEM supplemented with $10 \%$ FBS. When reaching confluency, each well was directly scratched with a $200 \mu$ l pipette tip. To detect the effect of irisin on the migration of U2OS and MG-63 cells, $100 \mathrm{ng} / \mathrm{ml}$ irisin was added to each well. After $24 \mathrm{~h}$ of incubation, the wound healing areas were photographed and the distance between the two cell edges was analyzed by ImageJ software.

In vitro invasion assay. The Transwell system was used to evaluate the effect of irisin on the invasion of U2OS and MG-63 osteosarcoma cells. The U2OS and MG-63 cells were cultured in Boyden chambers, with $8-\mu \mathrm{m}$ pore filter inserts, in 24 -well plates (Corning Costar, Corning, NY, USA). The pore inserts were pre-coated with Matrigel (BD Biosciences, San Jose, CA, USA) overnight. The U2OS and MG-63 cells ( $1 \times 10^{5}$ cells/well) were suspended in $100 \mu \mathrm{l}$ DMEM supplemented with $1 \%$ FBS and were added to the upper chamber. DMEM supplemented with $10 \%$ FBS and irisin $(100 \mathrm{ng} / \mathrm{ml})$ were added to the lower chamber. After $24 \mathrm{~h}$ of incubation, the cells that had attached to the lower surface were fixed with methanol and stained with $0.1 \%$ crystal violet. Then, 5 random high-power fields (magnification, $\mathrm{x} 200$ ) of each sample were chosen and counted to evaluate the average number of invasive cells.

RNA extraction and quantitative reverse transcription-PCR. Total RNA was extracted from the U2OS and MG-63 osteosarcoma cells using TRIzol reagent (Invitrogen Life Technologies) and then reverse transcribed to cDNA with the RevertAid First Strand cDNA Synthesis kit (Thermo Fisher Scientific, Inc.) according to the manufacturer's instructions. qPCR analysis was performed using a LightCycler (Bio-Rad Laboratories, Inc., Hercules, CA, USA). The primer sequences used for qPCR were as follows: E-cadherin forward, 5'-CGAGAGCTACACGTTCACGG-3' and reverse, 5'-GGGTGTCGAGGGAAAAATAGG-3'; N-cadherin forward, 5'-TTTGATGGAGGTCTCCTAACACC-3' and reverse, 5'-ACGTTTAACACGTTGGAAATGTG-3'; vimentin forward, 5'-TGCCGTTGAAGCTGCTAACTA-3' and reverse, 5'-CCAGAGGGAGTGAATCCAGATTA-3'; fibronectin forward, 5'-TCTCCTGCCTGGTACAGAATATGTAGT GAG-3' and reverse, 5'-GGTCGCAGCAACAACTTCCA GGT-3'; Snail forward, 5'-TCGGAAGCCTAACTACAG CGA-3' and reverse, 5'-AGATGAGCATTGGCAGCGAG-3'; 
MMP-2 forward, 5'-TTGATGGCATCGCTCAGATC-3' and reverse, 5'-TTGTCACGTGGCGTCACAGT-3'; MMP-9 forward, 5'-GACGCAGACATCGTCATCCA-3' and reverse, 5'-CACAACTCGTCATCGTCGAAA-3'; MMP-7 forward, 5'-GAGTGAGCTACAGTGGGAACA-3' and reverse, 5'-CTATGACGCGGGAGTTTAACAT-3'; GAPDH forward, 5'-GGAGCGAGATCCCTCCAAAAT-3' and reverse, 5'-GGCTGTTGTCATACTTCTCATGG-3'. Melting curves were assessed to confirm the specificity of the products generated for each set of primers. Following amplification, the $\Delta \Delta \mathrm{Ct}$ comparative method was used to normalize the relative levels of gene expression. The experiments were performed in triplicate.

Western blot analysis. After being treated with and without IL-6 and/or irisin, the U2OS and MG-63 osteosarcoma cells were collected and lysed. Total cell protein concentrations were assessed using the BCA protein assay kit (Pierce, Rockford, IL, USA). Equal protein from the cell lysates was loaded onto $12 \%$ SDS-PAGE. After electrophoresis, the proteins were transferred onto PVDF membranes (Millipore, Billerica, MA, USA) and then blocked with 5\% fat-free milk at room temperature for $1 \mathrm{~h}$ and incubated with primary antibodies overnight at $4^{\circ} \mathrm{C}$. Then, the membranes were washed with TBST and incubated with HRP-conjugated secondary antibodies for $1 \mathrm{~h}$ at room temperature. Immune complexes were detected with ECL reagents (Millipore, USA), and the blots were quantified by densitometric analysis using the Alpha Imager 2200.

Immunofluorescence staining. After being treated with and without IL-6 and/or irisin, the U2OS osteosarcoma cells were fixed with $4 \%$ paraformaldehyde, permeabilized with $0.5 \%$ Triton X-100 and then incubated with 5\% normal goat serum for $1 \mathrm{~h}$. Subsequently, the U2OS cells were incubated with rabbit anti-E-cadherin and rabbit anti-vimentin overnight at $4{ }^{\circ} \mathrm{C}$. This step was followed by incubation with Alexa Fluor 488-conjugated goat anti-rabbit $\operatorname{IgG}$ for $1 \mathrm{~h}$ at room temperature. Then the cells were incubated with 4',6-diamidino-2-phenylindole (DAPI)/PBS (1:5,000; Sigma-Aldrich) for $5 \mathrm{~min}$ at room temperature. Finally, the images were captured using a Nikon Eclipse 80i fluorescence microscope.

Statistical analysis. The data are expressed as the mean \pm standard deviation (SD). All of the experiments were repeated at least three times. A Student's t-test was used to compare differences between different groups. Comparisons among values of multiple groups were performed by one-way analysis of variance (ANOVA). Differences were considered to be statistically significant at $\mathrm{P}<0.05$.

\section{Results}

Irisin treatment inhibits the proliferation, migration and invasion of osteosarcoma cells. To explore the effect of irisin on the proliferation of osteosarcoma cells, an MTT assay was applied as previously described in Materials and methods. As shown in Fig. 1A, various doses $(0,25,50,100$ and $200 \mathrm{ng} / \mathrm{ml}$ ) of irisin were added into the cultured U2OS and MG-63 osteosarcoma cells. After $24 \mathrm{~h}$ of incubation, irisin significantly suppressed the viability of U2OS and MG-63 osteosarcoma cells at a concentration of $200 \mathrm{ng} / \mathrm{ml}$. After a 48-h incubation, irisin significantly inhibited the proliferation of U2OS and MG-63 osteosarcoma cells at a dose above $25 \mathrm{ng} / \mathrm{ml}(50,100$ and $200 \mathrm{ng} / \mathrm{ml})$. Therefore, irisin inhibited the proliferation of U2OS and MG-63 osteosarcoma cells in a dose- and time-dependent manner. Based on these results, we selected irisin at a concentration of $100 \mathrm{ng} / \mathrm{ml}$ for $24 \mathrm{~h}$ and conducted the following migration and invasion experiments, so as to eliminate the effect of irisin on U2OS and MG-63 osteosarcoma cell proliferation.

The wound healing assay was applied to detect the effect of irisin on the migration of osteosarcoma cells. The U2OS and MG-63 osteosarcoma cells were treated with $100 \mathrm{ng} / \mathrm{ml}$ irisin for $24 \mathrm{~h}$. As shown in Fig. 1B, the migration of U2OS and MG-63 cells was suppressed by $100 \mathrm{ng} / \mathrm{ml}$ irisin. The results of the wound healing assay revealed that the relative scratch width was significantly decreased when treated with irisin. To further explore the effect of irisin on osteosarcoma cell invasion, a Transwell assay was used. The U2OS and MG-63 cells were treated with $100 \mathrm{ng} / \mathrm{ml}$ irisin for $24 \mathrm{~h}$. As shown in Fig. 1C, the invasion of U2OS and MG-63 cells was inhibited by $100 \mathrm{ng} / \mathrm{ml}$ irisin. The results of the Transwell assay revealed that irisin may inhibit the invasive ability of osteosarcoma cells.

Irisin treatment reverses the IL-6-induced EMT in osteosarcoma cells. Evidence that EMT plays an important role in tumor invasion and metastasis have been well demonstrated $(42,43)$. In addition the role of IL- 6 in the induction of EMT has been established (44). To further explore the effect of irisin on osteosarcoma cell EMT, the expression of EMT markers (E-cadherin, N-cadherin, vimentin, fibronectin, MMP-2, MMP-7 and MMP-9) when treated with IL-6 (100 ng/ml) and irisin $(0,50$ and $100 \mathrm{ng} / \mathrm{ml})$ was detected using qPCR and western blot analysis. As illustrated in Fig. 2A and B, IL-6 inhibited E-cadherin protein expression and irisin restored the effect of IL-6 in a dose-dependent manner. The expression of N-cadherin, vimentin, fibronectin, MMP-2, MMP-7 and MMP-9 was upregulated when treated with IL-6 and irisin suppressed the effect in a dose-dependent manner. We also used immunofluorescence staining to evaluate the effect of irisin on the expression of EMT markers. After being treated with IL-6 $(100 \mathrm{ng} / \mathrm{ml})$ and irisin $(100 \mathrm{ng} / \mathrm{ml})$ for $24 \mathrm{~h}$, the U2OS cells were stained with $\mathrm{E}$-cadherin and vimentin and were analyzed by fluorescence microscopy. As shown in Fig. 2C, IL-6 downregulated E-cadherin expression and upregulated vimentin expression, while irisin effectively reversed the effect of IL-6. Collectively, these results indicated that irisin treatment prevented IL-6-induced EMT. Furthermore they revealed that the suppressive effect of irisin on osteosarcoma cell invasion and migration may be related to EMT.

Irisin inhibits IL-6-induced STAT3 phosphorylation in osteosarcoma cells. The MAPK and STAT3 signaling pathways play a crucial role in IL-6-induced cancer cell EMT (44-46). To further explore the potential mechanism of irisin on osteosarcoma cell EMT, the STAT3, ERK1/2 and $\mathrm{p} 38$ signaling pathways were detected. As shown in Fig. 3A, STAT3, ERK1/2 and p38 phosphorylation was upregulated while being treated with IL-6 (100 ng/ml). STAT3 phosphorylation was suppressed when 
A

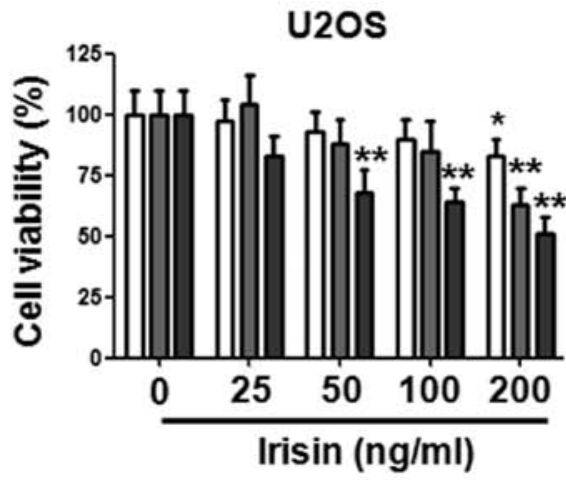

B

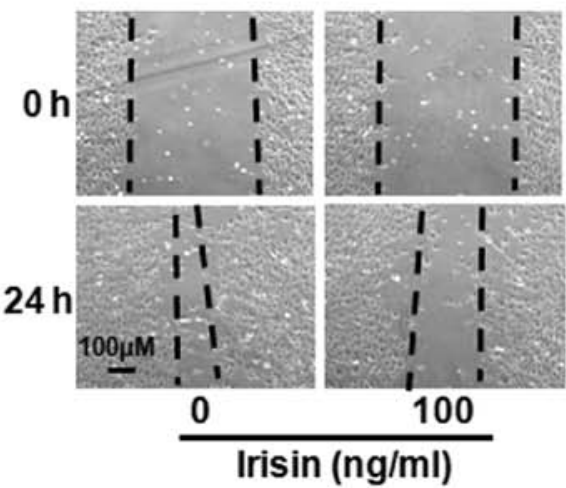

MG-63

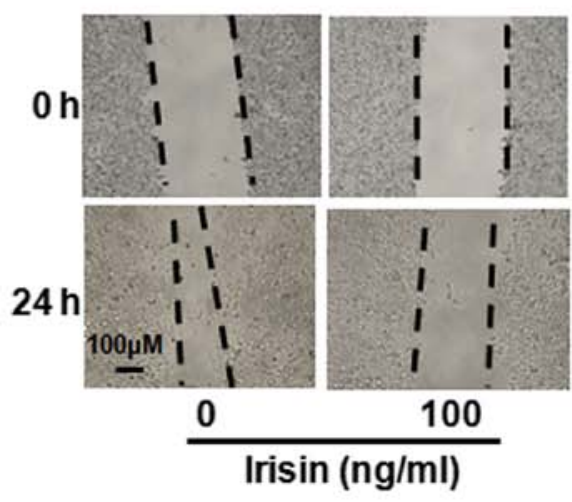

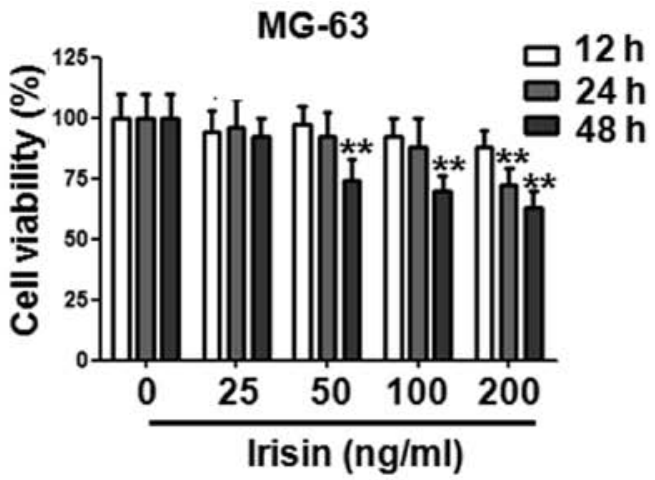

C

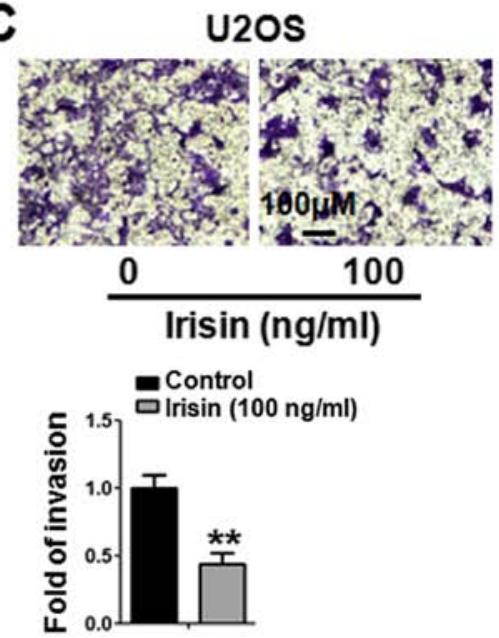

MG-63

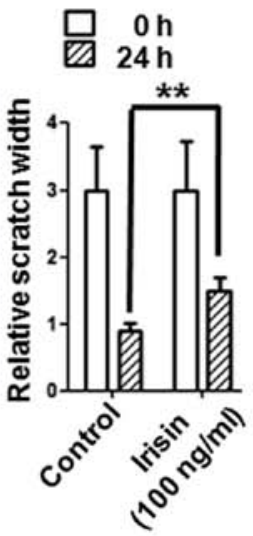

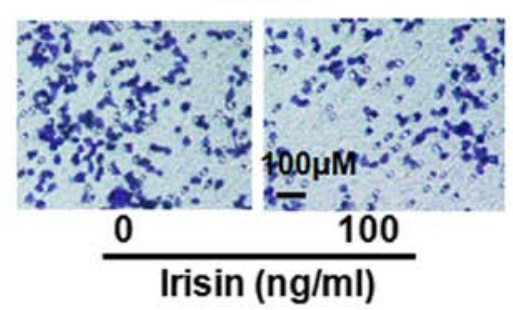

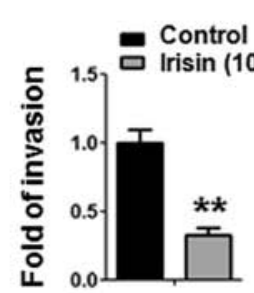

Figure 1. Irisin inhibits the proliferation, migration and invasion of U2OS and MG-63 osteosarcoma cells. (A) After treatment with irisin for various time-points (12, 24 and $48 \mathrm{~h})$ and at different concentration gradients $(0,25,50,100$ and $200 \mathrm{ng} / \mathrm{ml})$, the viability of the U2OS and MG-63 osteosarcoma cells was assessed by MTT assay. (B) The U2OS and MG-63 osteosarcoma cells were treated with irisin at a concentration of $100 \mathrm{ng} / \mathrm{ml}$ for $24 \mathrm{~h}$ and then assessed by a wound healing assay. (C) The U2OS and MG-63 osteosarcoma cells were treated with irisin at a concentration of $100 \mathrm{ng} / \mathrm{ml}$ for $24 \mathrm{~h}$. The effect of irisin on the invasion of U2OS and MG-63 osteosarcoma cells was examined by a Transwell assay. The bar graph represents the results of three independent experiments. ${ }^{*} \mathrm{P}<0.05,{ }^{* * *} \mathrm{P}<0.01$ vs. the control group. The results represent the mean $\pm \mathrm{SD}$ from three independent experiments. MTT, 3-(4,5-dimethylthiazol2-yl)-2,5-diphenyltetrazolium bromide.

treated with irisin $(100 \mathrm{ng} / \mathrm{ml})$. The suppression was statistically significant, as quantified by densitometry (Fig. 3B). However, irisin did not change the phosphorylation of ERK1/2 and p38. These results revealed that the STAT3 signaling pathway may be involved in irisin-mediated osteosarcoma cell EMT.

The STAT3 pathway is involved in irisin-modulated EMT in osteosarcoma cells. To further demonstrate whether STAT3 is involved in irisin-regulated osteosarcoma cell EMT, we selected WP1066 (a STAT3 inhibitor) to block STAT3 phosphorylation (Fig. 4A). As shown in Fig. 4B and C, at the mRNA and protein level, the suppression of STAT3 phosphorylation with WP1066 $(10 \mu \mathrm{M})$ enhanced the expression of E-cadherin and further downregulated the expression of some EMT markers (N-cadherin, fibronectin, MMP-2 and MMP-7) which were induced by irisin. These results revealed that irisin-mediated 


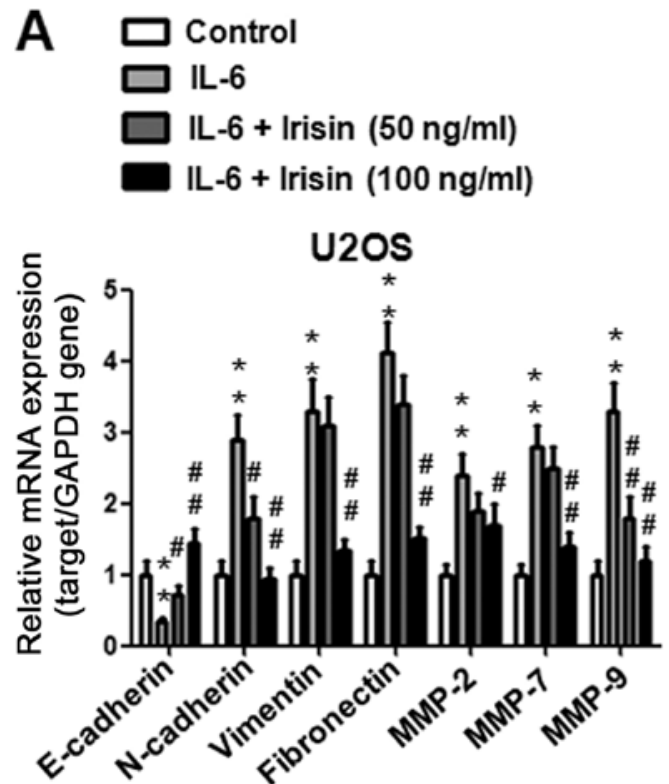

MG-63

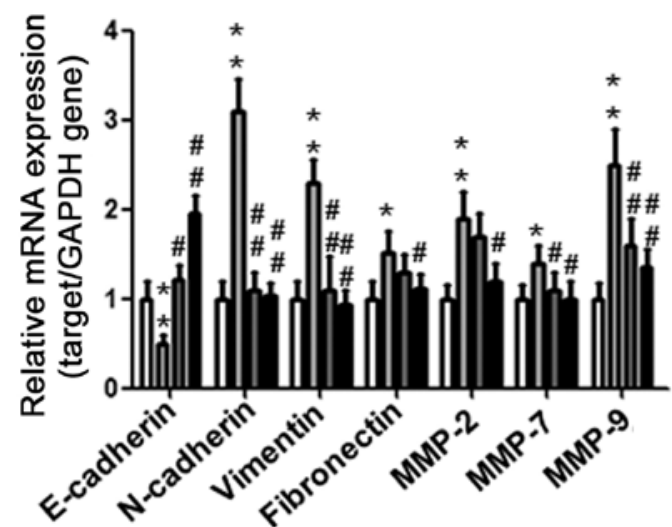

B

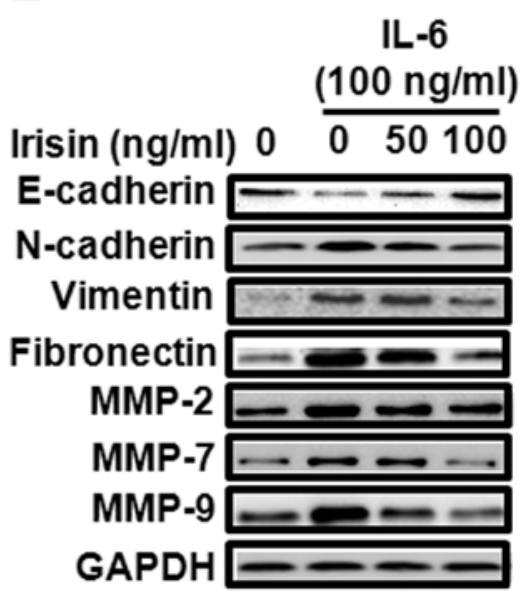

MG-63

IL-6

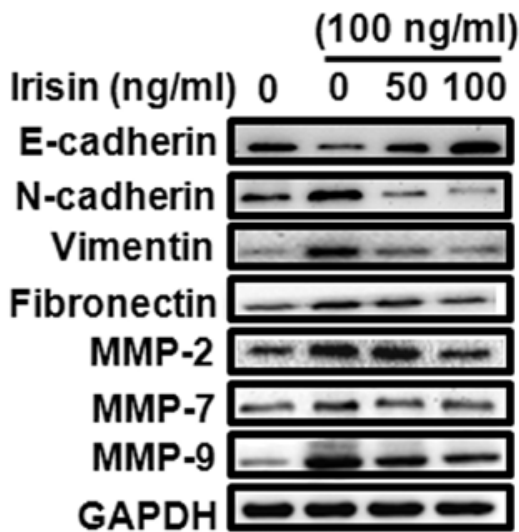

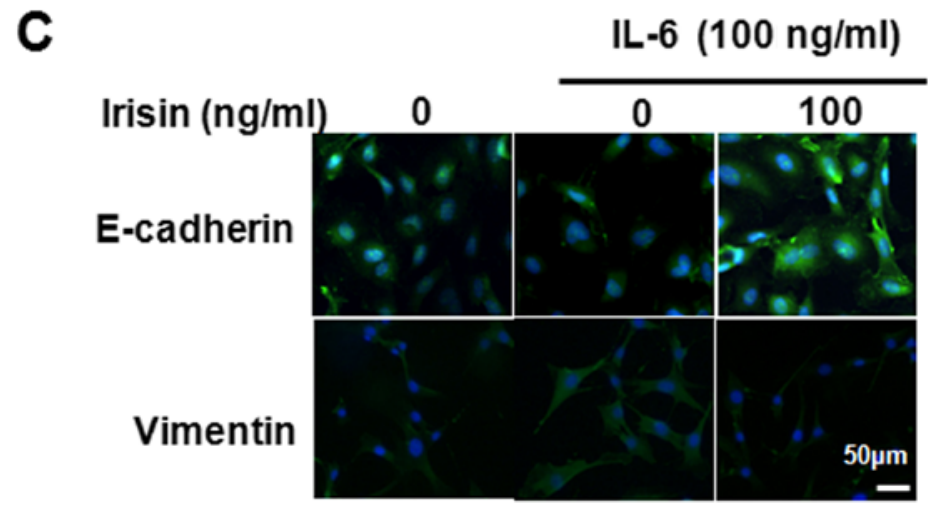

Figure 2. Irisin treatment reverses IL-6-mediated EMT in U2OS and MG-63 osteosarcoma cells. (A and B) The U2OS and MG-63 osteosarcoma cells were treated with or without IL-6 (100 ng/ml) and/or irisin at the indicated concentrations $(0,50$ and $100 \mathrm{ng} / \mathrm{ml}) \mathrm{for} 24 \mathrm{~h}$ and the expression level of E-cadherin, $\mathrm{N}$-cadherin, vimentin, fibronectin, MMP-2, MMP-7 and MMP-9 mRNA and the protein expression was examined by qPCR analysis and western blot analysis (C) The U2OS osteosarcoma cells were treated with or without IL-6 (100 ng/ml) and/or irisin at the indicated concentrations (100 ng/ml) for $24 \mathrm{~h}$ and the expression of E-cadherin and vimentin was detected by immunofluorescence staining. ${ }^{*} \mathrm{P}<0.05,{ }^{* * *} \mathrm{P}<0.01$ vs. the control group; ${ }^{\#} \mathrm{P}<0.05$, ${ }^{\# \#} \mathrm{P}<0.01$, compared with the cells treated with IL-6. Data shown are expressed as the means \pm SD from three independent experiments. EMT, epithelial-mesenchymal transition.

osteosarcoma cell EMT may be STAT3-dependent. Blockade of STAT3 activation could further enhance irisin-mediated osteosarcoma cell EMT.

Irisin inhibits IL-6-induced Snail upregulation via the STAT3 pathway in osteosarcoma cells. Evidence has established the expression and activation of Snail transcriptional factor during EMT in E-cadherin suppression and cancer progression (47). In the present study we further explored the effects of irisin on the IL-6-induced Snail expression and the relationship between STAT3 and Snail. Firstly, U2OS and MG-63 osteosarcoma cells were treated with IL-6 $(100 \mathrm{ng} / \mathrm{ml})$ and irisin $(0,50$ and $100 \mathrm{ng} / \mathrm{ml})$ for $24 \mathrm{~h}$ and the expression of Snail was detected using qPCR and western 


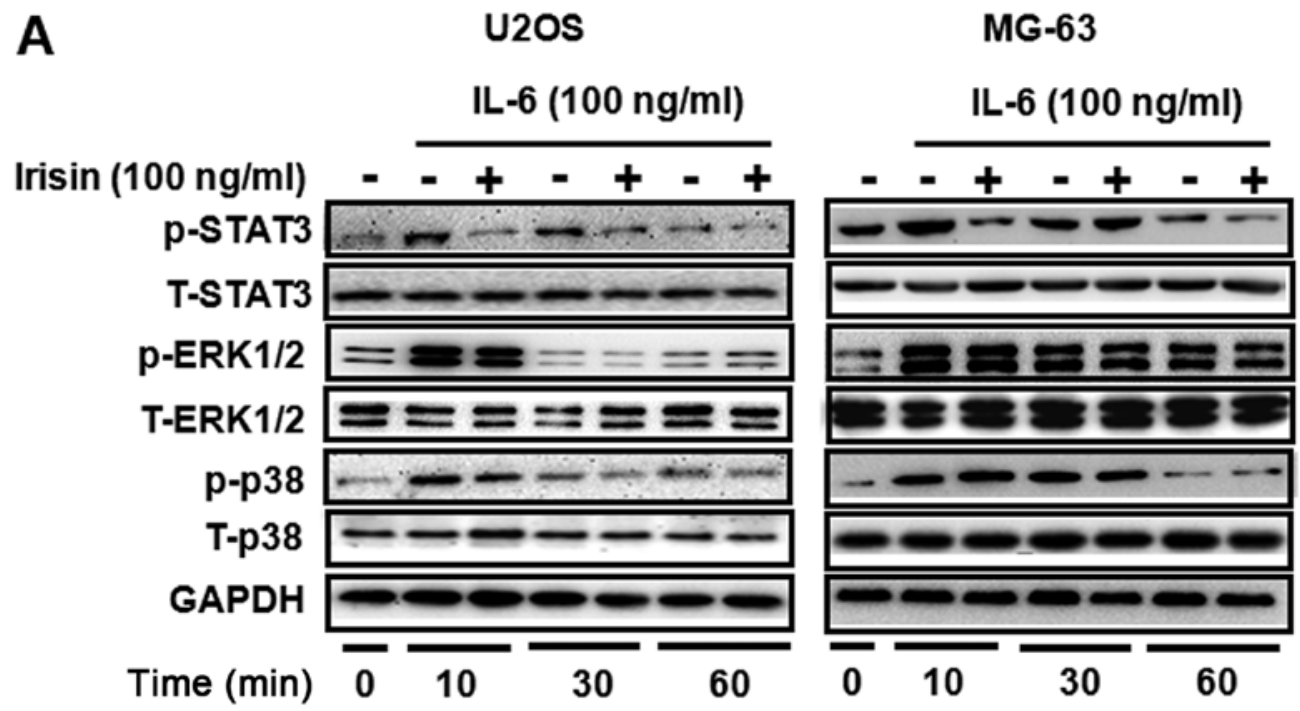

B
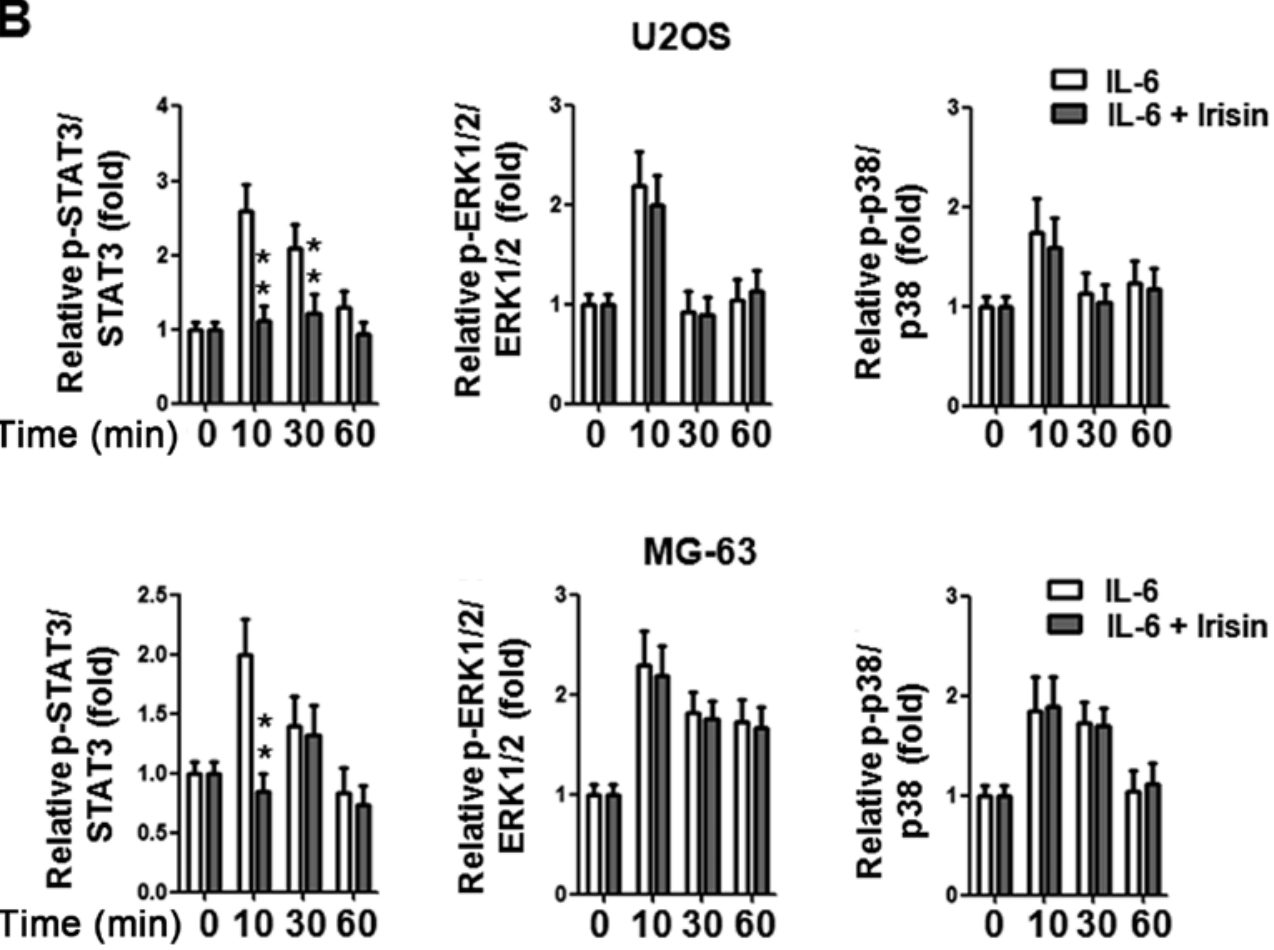

Figure 3. Irisin suppresses STAT3 phosphorylation induced by IL-6 stimulation. The U2OS and MG-63 osteosarcoma cells treated with or without IL-6 (100 ng/ml) and/or irisin (100 ng/ml) at indicated time-points. (A) Phospho- and total STAT3, ERK1/2 and p38 levels in cell lysates were detected by western blot analysis. (B) Statistical analysis of the western blotting results. ${ }^{*} \mathrm{P}<0.05,{ }^{* *} \mathrm{P}<0.01$ vs. the cells treated with IL-6. Data shown are expressed as the means \pm SD from three independent experiments.

blot analysis. As shown in Fig. 5A and B, IL-6 induced Snail mRNA and protein expression and irisin inhibited the effect in a dose-dependent manner. Then, to further evaluate whether STAT3 participated in irisin-regulated Snail expression, U2OS and MG-63 osteosarcoma cells were also pretreated with WP1066 for 30 min before IL-6 and irisin were added. As displayed in Fig. 5C and D, inhibition of STAT3 phosphorylation with WP1066 further suppressed the mRNA and protein expression of Snail which was induced by irisin. These results indicated the crucial role of STAT3 in regulating Snail expression and irisin inhibition of the expression of Snail via the STAT3 pathway. Collectively, these results demonstrated that the suppression of metastasis by irisin was mediated by the inhibition of EMT via the STAT3/Snail signaling pathway in osteosarcoma cells.

\section{Discussion}

The current study revealed several new findings about irisin. Irisin can inhibit the proliferation, migration and invasion of osteosarcoma cells. Irisin can also suppress IL-6-induced EMT in osteosarcoma cells. The beneficial effects of irisin in the EMT of osteosarcoma cells were observed through the STAT3/Snail signaling pathway.

Recently, much attention has been paid on the potential benefits of exercise in chronic metabolic diseases, 
A

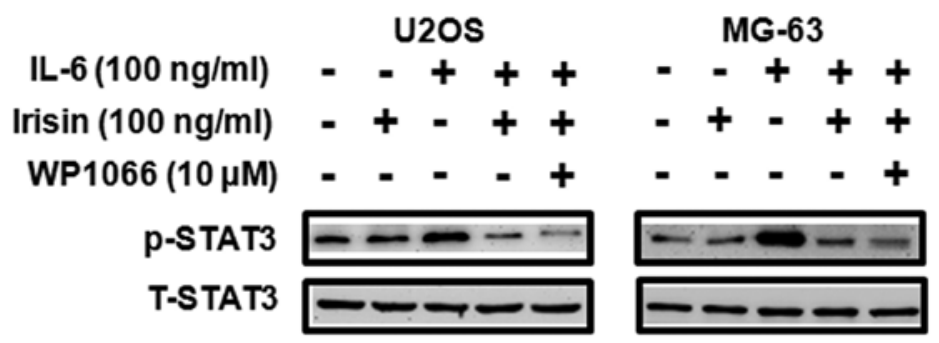

B

U2OS

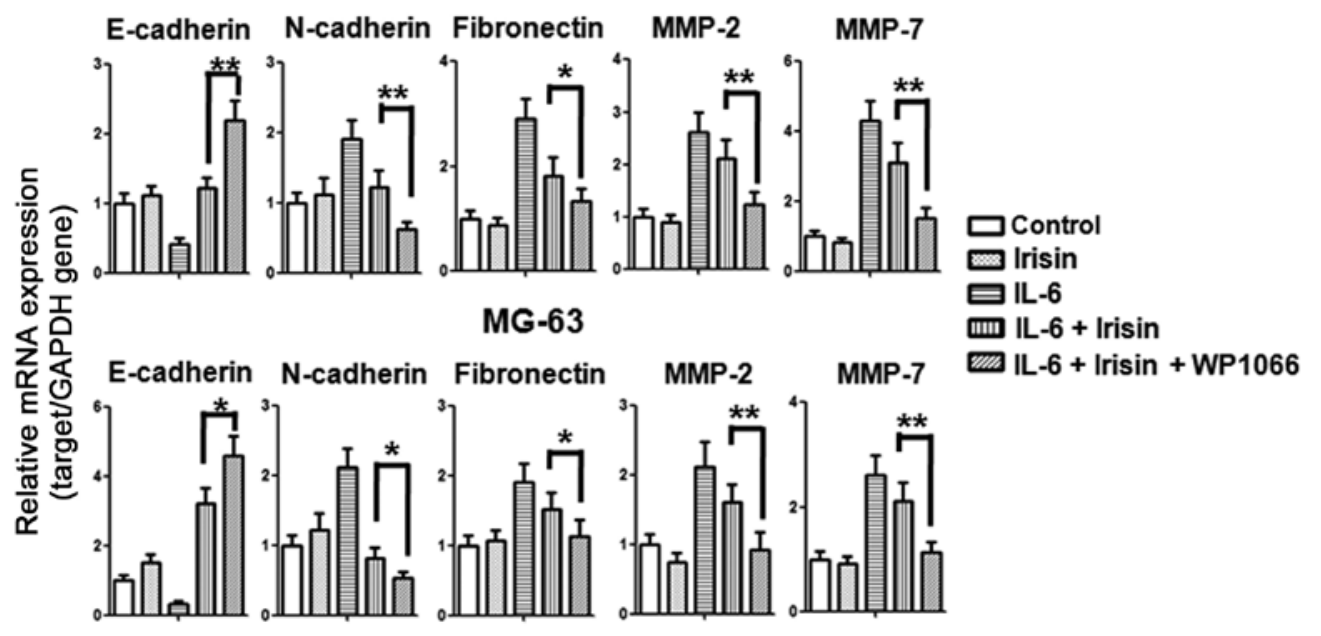

C

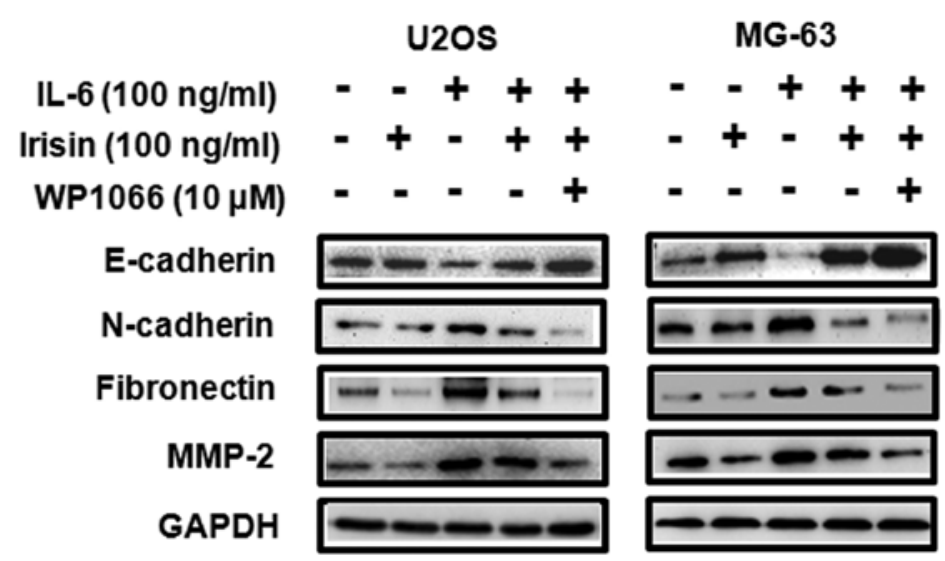

Figure 4. Inhibition of STAT3 signaling enhances the effect of irisin on IL-6-induced EMT in U2OS and MG-63 osteosarcoma cells. The U2OS and MG-63 osteosarcoma cells were pretreated with the STAT3 inhibitor WP1066 (10 $\mu \mathrm{M})$ for $30 \mathrm{~min}$ followed by IL-6 (100 ng/ml) and/or irisin (100 ng/ml) treatment for $24 \mathrm{~h}$. (A) Western blot analysis of the phosphorylated STAT3 and total STAT3 protein expression. (B) qPCR analysis of the mRNA expression of E-cadherin, N-cadherin, fibronectin, MMP-2, and MMP-7. (C) Western blot analysis of the protein expression of E-cadherin, N-cadherin, fibronectin and MMP-2. ${ }^{*} \mathrm{P}<0.05,{ }^{* *} \mathrm{P}<0.01$, compared with the cells treated with IL-6 and irisin. Data shown are expressed as the means \pm SD from three independent experiments. EMT, epithelial-mesenchymal transition.

cardiovascular diseases and cancer (1-4). As a novel discovered myokine, irisin is released from the skeletal muscles following exercise (9). Previous studies have demonstrated that irisin modulated body energy expenditure by promoting brown adipocyte thermogenesis in mice $(9,10)$. Meanwhile, recent studies have revealed the beneficial effect of irisin in neuronal cells (11), adipocytes (12), osteoblasts $(13,14)$, as well as vascular cells, such as endothelial cells $(15,18)$ and smooth muscle cells (17). Furthermore, recent studies revealed the direct suppressive effect of irisin on malignant breast and lung cancer cell proliferation and migration $(23,24)$. In addition with other findings concerning the expression of irisin in various cancer tissues (20-22), these findings revealed the close association between irisin and cancer. The present study is the first to investigate the role of irisin on osteosarcoma cell migration and invasion, as well as its detailed molecular mechanisms. Firstly, we evaluated the effect of irisin on the proliferation of osteosarcoma cells. We found that $100 \mathrm{ng} / \mathrm{ml}$ of irisin significantly suppressed cell proliferation after $48 \mathrm{~h}$, while there was no significant effect before $24 \mathrm{~h}$. We selected $24 \mathrm{~h}$ as the stimulation time-point and $100 \mathrm{ng} / \mathrm{ml}$ as the stimulation concentration in the following migration and invasion experiments, so that the influence of cell proliferation was excluded. Our study indicated that irisin effectively 

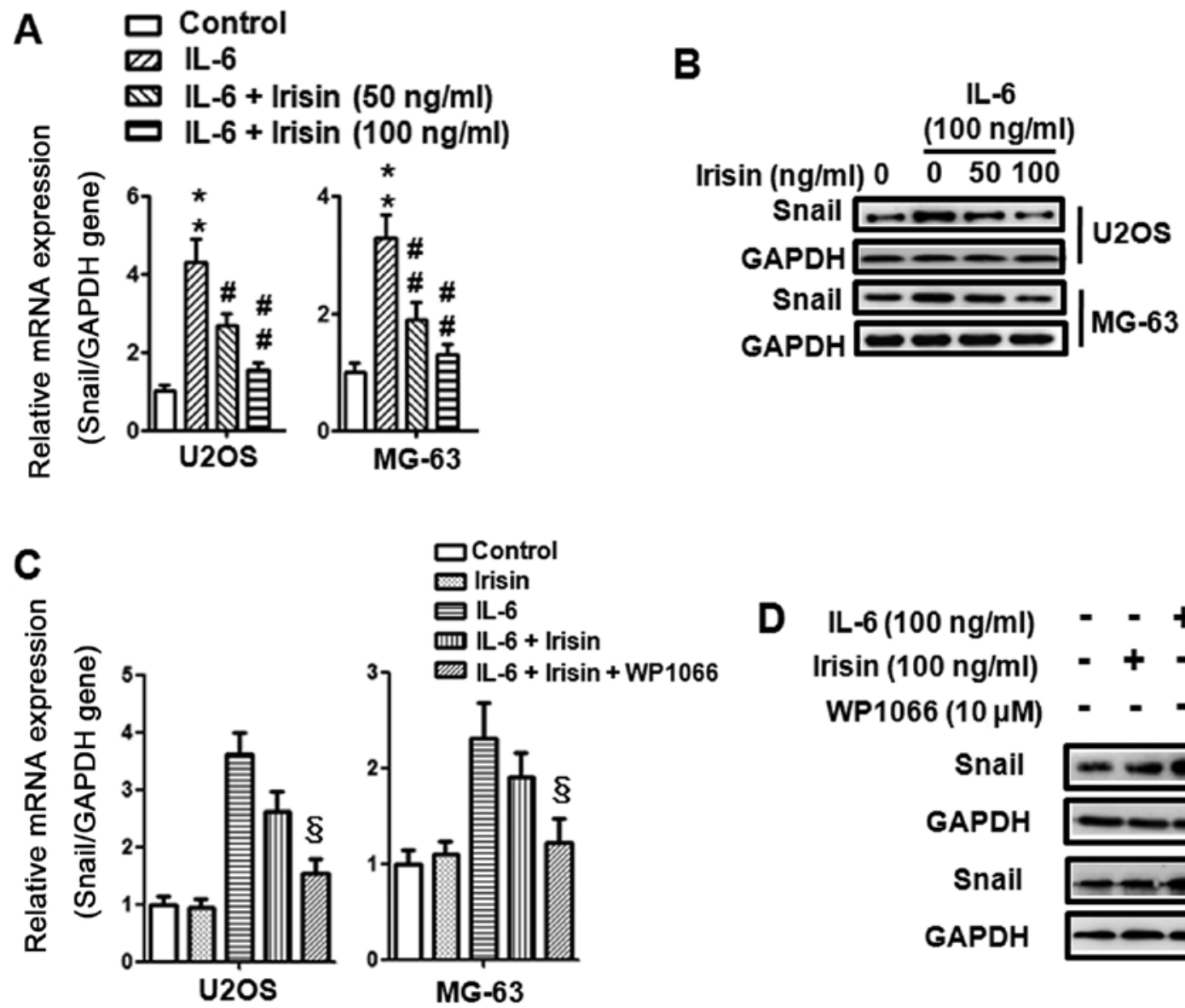

Figure 5. STAT3 is involved in irisin-mediated Snail expression induced by IL-6 stimulation in U2OS and MG-63 osteosarcoma cells. (A and B) The U2OS and MG-63 osteosarcoma cells were treated with or without IL-6 $(100 \mathrm{ng} / \mathrm{ml})$ and/or irisin at indicated concentrations $(0,50 \mathrm{and} 100 \mathrm{ng} / \mathrm{ml})$ for $24 \mathrm{~h}$ and the expression level of Snail mRNA and protein expression was examined by qPCR analysis and western blot analysis. (C and D) The U2OS and MG-63 osteosarcoma cells were pretreated with the STAT3 inhibitor WP1066 $(10 \mu \mathrm{M})$ for $30 \mathrm{~min}$ followed by IL-6 (100 ng/ml) and $/ \mathrm{or}$ irisin (100 ng/ml) treatment for $24 \mathrm{~h}$, and then the mRNA and protein expression of Snail were assessed by qPCR analysis and western blotting assay. ${ }^{*} \mathrm{P}<0.05,{ }^{* *} \mathrm{P}<0.01 \mathrm{vs}$. the control group; ${ }^{\#} \mathrm{P}<0.05,{ }^{\# \#} \mathrm{P}<0.01$, compared with the cells treated with IL-6. ${ }^{\S} \mathrm{P}<0.05,{ }^{\$} \mathrm{P}<0.01$, compared with the cells treated with IL-6 and irisin. Data shown are expressed as the means $\pm \mathrm{SD}$ from three independent experiments.

suppressed the migration and invasion of osteosarcoma cells. These finding demonstrated the anti-metastatic property of irisin.

Metastasis is considered as the primary cause of mortality in most cancer patients. Studies have well established that EMT is a distinctive phenotypic switch by which epithelial cells lose their polarity and acquire the invasive properties of mesenchymal cells in the process of metastasis (48). Furthermore, studies have demonstrated that IL-6 could induce EMT in pancreatic, lung, hepatocellular and colorectal cancer cells (32-35). In the present study, we revealed the role of irisin in IL-6-induced EMT in osteosarcoma cells. Our study found that irisin could significantly reverse the IL-6-induced downregulation of the epithelial marker (E-cadherin) and upregulation of mesenchymal markers (N-cadherin, vimentin, fibronectin, MMP-2, MMP-7 and MMP-9) in osteosarcoma cells. Therefore, for the first time, our study demonstrated that irisin could effectively reverse the IL-6-induced EMT of osteosarcoma cells.

IL-6, a major cytokine in the tumor microenvironment, has long been documented in tumorigenesis in the regulation of proliferation, apoptosis, angiogenesis and metastasis of various types of cancer cells (49). Studies have demonstrated that the
MAPK and STAT3 pathways were involved in IL-6-regulation of various cancer cell functions, such as proliferation and metastasis (30,36-38). Notably, STAT3 has been documented to play a crucial role in IL-6-induced EMT $(33,38)$. In the present study, the expression of STAT3, ERK1/2 and p38 in osteosarcoma cells was evaluated. We found that the expression of phosphor-STAT3, phosphor-ERK1/2 and phosphor-p38 was increased when treated with IL-6, while only the phosphorylation of STAT3 was inhibited by irisin treatment. To further examine whether the STAT3 pathway was involved in irisin-regulated EMT in osteosarcoma cells induced by IL-6, STAT3 was blocked using WP1066 (a STAT3 inhibitor). We found that inhibition of the STAT3 pathway significantly further enhanced irisin-mediated EMT of osteosarcoma cells. These results demonstrated that STAT3 is essential for IL-6-induced EMT in osteosarcoma cells. These results also revealed that the effect of irisin may be STAT3-dependent.

Finally, we explored the effect of irisin and STAT3 on the transcription factor Snail. Snail, a zinc finger transcription factor, has been demonstrated to modulate the transcriptional suppression of E-cadherin (50). Previous studies revealed that the expression of Snail was modulated by STAT3 (51). Another study further demonstrated the crucial role of the 
STAT3/Snail pathway in regulating EMT in hepatocellular carcinoma cells (52). In the current study, we found that irisin suppressed the upregulation of Snail which was induced by IL-6, while blockade of STAT3 further downregulated irisinmediated Snail expression in osteosarcoma cells. These results revealed that STAT3 was upstream of Snail. Furthermore, the aforementioned results demonstrated that irisin suppressed the expression of Snail via the STAT3 pathway.

In conclusion, for the first time, our study revealed that irisin suppressed the migration and invasion of osteosarcoma cells. Moreover, irisin reversed the EMT induced by IL-6 in osteosarcoma cells. Finally, the inhibitory role of irisin in IL-6-induced EMT was modulated via the STAT3/Snail pathway. Collectively, irisin reversed IL-6-induced EMT of osteosarcoma cells through the STAT3/Snail signaling pathway. Thus, our research indicated that irisin is a promising agent in osteosarcoma treatment. Therefore further in vivo research needs to be performed.

\section{References}

1. De Sousa SM and Norman RJ: Metabolic syndrome, diet and exercise. Best Pract Res Clin Obstet Gynaecol 37: 140-151, 2016.

2. Francesconi C, Lackinger C, Weitgasser R, Haber P and Niebauer J: Physical activity and exercise training in the prevention and therapy of type 2 diabetes mellitus. Wien Klin Wochenschr 128: S141-S145, 2016 (In German).

3. Endes S: Physical activity reduces cardiovascular disease risk in older adults. Evid Based Med 21: 191, 2016.

4. Navigante A and Morgado PC: Does physical exercise improve quality of life of advanced cancer patients? Curr Opin Support Palliat Care 10: 306-309, 2016.

5. Nyrop KA, Deal AM, Williams GR, Guerard EJ, Pergolotti M and Muss HB: Physical activity communication between oncology providers and patients with early-stage breast, colon or prostate cancer. Cancer 122: 470-476, 2007.

6. Michaels C: The importance of exercise in lung cancer treatment Transl Lung Cancer Res 5: 235-238, 2016.

7. Lin KY, Frawley HC, Denehy L, Feil D and Granger CL: Exercise interventions for patients with gynaecological cancer: A systematic review and meta-analysis. Physiotherapy 102: 309-319, 2016.

8. Karstoft K and Pedersen BK: Skeletal muscle as a gene regulatory endocrine organ. Curr Opin Clin Nutr Metab Care 19: 270-275, 2016.

9. Boström P, Wu J, Jedrychowski MP, Korde A, Ye L, Lo JC, Rasbach KA, Boström EA, Choi JH, Long JZ, et al: A PGC1- $\alpha$ dependent myokine that drives brown-fat-like development of white fat and thermogenesis. Nature 481: 463-468, 2012.

10. Wu J, Boström P, Sparks LM, Ye L, Choi JH, Giang AH, Khandekar M, Virtanen KA, Nuutila P, Schaart G, et al: Beige adipocytes are a distinct type of thermogenic fat cell in mouse and human. Cell 150: 366-376, 2012.

11. Moon HS, Dincer F and Mantzoros CS: Pharmacological concentrations of irisin increase cell proliferation without influencing markers of neurite outgrowth and synaptogenesis in mouse H19-7 hippocampal cell lines. Metabolism 62: 1131-1136, 2013.

12. Huh JY, Dincer F, Mesfum E and Mantzoros CS: Irisin stimulates muscle growth-related genes and regulates adipocyte differentiation and metabolism in humans. Int $\mathbf{J}$ Obes (Lond) 38: $1538-1544,2014$

13. Qiao X, Nie Y, Ma Y, Chen Y, Cheng R, Yin W, Hu Y, Xu W and $\mathrm{Xu}$ L: Irisin promotes osteoblast proliferation and differentiation via activating the MAP kinase signaling pathways. Sci Rep 6: 18732,2016

14. Colaianni G, Cuscito C, Mongelli T, Pignataro P, Buccoliero C, Liu P, Lu P, Sartini L, Di Comite M, Mori G, et al: The myokine irisin increases cortical bone mass. Proc Natl Acad Sci USA 112: 12157-12162, 2015.

15. Song H, Wu F, Zhang Y, Zhang Y, Wang F, Jiang M, Wang Z, Zhang M, Li S, Yang L, et al: Irisin promotes human umbilical vein endothelial cell proliferation through the ERK signaling pathway and partly suppresses high glucose-induced apoptosis. PLoS One 9: e110273, 2014.
16. Lu J, Xiang G, Liu M, Mei W, Xiang L and Dong J: Irisin protects against endothelial injury and ameliorates atherosclerosis in apolipoprotein E-Null diabetic mice. Atherosclerosis 243: 438-448, 2015.

17. Song H, Xu J, Lv N, Zhang Y, Wu F, Li H, Shao L, Mu Q, Wang F, Tang D, et al: Irisin reverses platelet derived growth factorBB-induced vascular smooth muscle cells phenotype modulation through STAT3 signaling pathway. Biochem Biophys Res Commun 479: 139-145, 2016.

18. Zhang Y, Song H, Zhang Y, Wu F, Mu Q, Jiang M, Wang F, Zhang W, Li L, Shao L, et al: Irisin inhibits atherosclerosis by promoting endothelial proliferation through microRNA126-5p. J Am Heart Assoc 5: 5, 2016.

19. Provatopoulou X, Georgiou GP, Kalogera E, Kalles V, Matiatou MA, Papapanagiotou I, Sagkriotis A, Zografos GC and Gounaris A: Serum irisin levels are lower in patients with breast cancer: Association with disease diagnosis and tumor characteristics. BMC Cancer 15: 898, 2015.

20. Aydin S, Kuloglu T, Ozercan MR, Albayrak S, Aydin S, Bakal U, Yilmaz M, Kalayci M, Yardim M, Sarac M, et al: Irisin immunohistochemistry in gastrointestinal system cancers. Biotech Histochem 91: 242-250, 2016.

21. Us Altay D, Keha EE, Ozer Yaman S, Ince I, Alver A, Erdogan B, Canpolat S, Cobanoglu U and Mentese A: Investigation of the expression of irisin and some cachectic factors in mice with experimentally induced gastric cancer. QJM 109: 785-790, 2016.

22. Kuloglu T, Celik O, Aydin S, Hanifi Ozercan I, Acet M, Aydin Y, Artas G, Turk A, Yardim M, Ozan G, et al: Irisin immunostaining characteristics of breast and ovarian cancer cells. Cell Mol Biol (Noisy-le-grand) 62: 40-44, 2016.

23. Gannon NP, Vaughan RA, Garcia-Smith R, Bisoffi M and Trujillo KA: Effects of the exercise-inducible myokine irisin on malignant and non-malignant breast epithelial cell behavior in vitro. Int J Cancer 136: 197-202, 2015.

24. Shao L, Li H, Chen J, Song H, Zhang Y, Wu F, Wang W, Zhang W, Wang F, Li H and Tang D: Irisin suppresses the migration, proliferation, and invasion of lung cancer cells via inhibition of epithelial-to-mesenchymal transition. Biochem Biophys Res Commun 485: 598-605, 2017.

25. Mirabello L, Troisi RJ and Savage SA: Osteosarcoma incidence and survival rates from 1973 to 2004: Data from the Surveillance, Epidemiology, and End Results Program. Cancer 115: 1531-1543, 2009

26. Gaffney R, Unni KK, Sim FH, Slezak JM, Esther RJ and Bolander ME: Follow-up study of long-term survivors of osteosarcoma in the prechemotherapy era. Hum Pathol 37: 1009-1014, 2006.

27. Vuoriluoto K, Haugen H, Kiviluoto S, Mpindi JP, Nevo J, Gjerdrum C, Tiron C, Lorens JB and Ivaska J: Vimentin regulates EMT induction by Slug and oncogenic H-Ras and migration by governing Axl expression in breast cancer. Oncogene 30: 1436-1448, 2011.

28. Rosano L, Cianfrocca R, Spinella F, Di Castro V, Nicotra MR, Lucidi A, Ferrandina G, Natali PG and Bagnato A: Acquisition of chemoresistance and EMT phenotype is linked with activation of the endothelin A receptor pathway in ovarian carcinoma cells. Clin Cancer Res 17: 2350-2360, 2011.

29. Davidson B, Tropé CG and Reich R: Epithelial-mesenchymal transition in ovarian carcinoma. Front Oncol 2: 33, 2012.

30. Tu B, Du L, Fan QM, Tang Z and Tang TT: STAT3 activation by IL-6 from mesenchymal stem cells promotes the proliferation and metastasis of osteosarcoma. Cancer Lett 325: 80-88, 2012.

31. Tzeng HE, Tsai CH, Chang ZL, Su CM, Wang SW, Hwang WL and Tang $\mathrm{CH}$ : Interleukin-6 induces vascular endothelial growth factor expression and promotes angiogenesis through apoptosis signal-regulating kinase 1 in human osteosarcoma. Biochem Pharmacol 85: 531-540, 2013.

32. Chen J, Wang S, Su J, Chu G, You H, Chen Z, Sun H, Chen B and Zhou M: Interleukin-32 $\alpha$ inactivates JAK2/STAT3 signaling and reverses interleukin-6-induced epithelial-mesenchymal transition, invasion, and metastasis in pancreatic cancer cells. Onco Targets Ther 9: 4225-4237, 2016.

33. Meng J, Zhang XT, Liu XL, Fan L, Li C, Sun Y, Liang XH, Wang JB, Mei QB, Zhang F, et al: WSTF promotes proliferation and invasion of lung cancer cells by inducing EMT via PI3K/Akt and IL-6/STAT3 signaling pathways. Cell Signal 28: 1673-1682, 2016. 
34. Wu J, Zhang J, Shen B, Yin K, Xu J, Gao W and Zhang L: Long noncoding RNA lncTCF7, induced by IL-6/STAT3 transactivation, promotes hepatocellular carcinoma aggressiveness through epithelial-mesenchymal transition. J Exp Clin Cancer Res 34: 116, 2015.

35. Liu H, Ren G, Wang T, Chen Y, Gong C, Bai Y, Wang B, Qi H, Shen J, Zhu L, et al: Aberrantly expressed Fra-1 by IL-6/STAT3 transactivation promotes colorectal cancer aggressiveness through epithelial-mesenchymal transition. Carcinogenesis 36 : 459-468, 2015.

36. Dou L, Wang S, Sui X, Meng X, Shen T, Huang X, Guo J, Fang W, Man Y, Xi J and Li J: MiR-301a mediates the effect of IL-6 on the AKT/GSK pathway and hepatic glycogenesis by regulating PTEN expression. Cell Physiol Biochem 35: 1413-1424, 2015.

37. Che Q, Liu BY, Wang FY, He YY, Lu W, Liao Y, Gu W and Wan XP: Interleukin 6 promotes endometrial cancer growth through an autocrine feedback loop involving ERK-NF- $\mathrm{B}$ signaling pathway. Biochem Biophys Res Commun 446: 167-172, 2014.

38. Wu YS, Chung I, Wong WF, Masamune A, Sim MS and Looi CY: Paracrine IL-6 signaling mediates the effects of pancreatic stellate cells on epithelial-mesenchymal transition via Stat3/Nrf2 pathway in pancreatic cancer cells. Biochim Biophys Acta 1861: 296-306, 2016

39. Pan J, Lee Y, Zhang Q, Xiong D, Wan TC, Wang Y and You M: Honokiol decreases lung cancer metastasis through inhibition of the STAT3 signaling pathway. Cancer Prev Res (Phila) 10 133-141, 2016

40. Xue D, Yang Y, Liu Y, Wang P, Dai Y, Liu Q, Chen L, Shen J, $\mathrm{Ju} \mathrm{H}, \mathrm{Li}$ Y, et al: MicroRNA-206 attenuates the growth and angiogenesis in non-small cell lung cancer cells by blocking the 14-3-3ร/STAT3/HIF-1 $\alpha /$ VEGF signaling. Oncotarget 7 : 79805-79813,2016.

41. Yuan H, Kajiyama H, Ito S, Yoshikawa N, Hyodo T, Asano E, Hasegawa H, Maeda M, Shibata K, Hamaguchi M, et al: ALX1 induces snail expression to promote epithelial-to-mesenchymal transition and invasion of ovarian cancer cells. Cancer Res 73 1581-1590, 2013

42. Mitra A, Mishra L and Li S: EMT, CTCs and CSCs in tumor relapse and drug-resistance. Oncotarget 6: 10697-10711, 2015.
43. Qureshi R, Arora H and Rizvi MA: EMT in cervical cancer: Its role in tumour progression and response to therapy. Cancer Lett 356 (2 Pt B): 321-331, 2015.

44. Wu D, Cheng J, Sun G, Wu S, Li M, Gao Z, Zhai S, Li P, Su D and Wang X: p70S6K promotes IL-6-induced epithelial-mesenchymal transition and metastasis of head and neck squamous cell carcinoma. Oncotarget 7: 36539-36550, 2016.

45. Lee SO, Yang X, Duan S, Tsai Y, Strojny LR, Keng P and Chen Y: IL-6 promotes growth and epithelial-mesenchymal transition of $\mathrm{CD}_{133^{+}}$cells of non-small cell lung cancer. Oncotarget 7: 6626-6638, 2016.

46. Bharti R, Dey G and Mandal M: Cancer development, chemoresistance, epithelial to mesenchymal transition and stem cells: A snapshot of IL-6 mediated involvement. Cancer Lett 375: 51-61, 2016.

47. Papiewska-Pająk I, Kowalska MA and Boncela J: Expression and activity of SNAIL transcription factor during epithelial to mesenchymal transition (EMT) in cancer progression. Postepy Hig Med Dosw (Online) 70: 968-980, 2016.

48. Turley EA, Veiseh M, Radisky DC and Bissell MJ: Mechanisms of disease: Epithelial-mesenchymal transition - does cellular plasticity fuel neoplastic progression? Nat Clin Pract Oncol 5: 280-290, 2008

49. Kumari N, Dwarakanath BS, Das A and Bhatt AN: Role of interleukin-6 in cancer progression and therapeutic resistance. Tumour Biol 37: 11553-11572, 2016.

50. Cano A, Pérez-Moreno MA, Rodrigo I, Locascio A, Blanco MJ, del Barrio MG, Portillo F and Nieto MA: The transcription factor snail controls epithelial-mesenchymal transitions by repressing E-cadherin expression. Nat Cell Biol 2: 76-83, 2000.

51. Saitoh M, Endo K, Furuya S, Minami M, Fukasawa A, Imamura T and Miyazawa K: STAT3 integrates cooperative Ras and TGF- $\beta$ signals that induce Snail expression. Oncogene 35: 1049-1057, 2016.

52. Fu XT, Dai Z, Song K, Zhang ZJ, Zhou ZJ, Zhou SL, Zhao YM, Xiao YS, Sun QM, Ding ZB, et al: Macrophage-secreted IL-8 induces epithelial-mesenchymal transition in hepatocellular carcinoma cells by activating the JAK2/STAT3/Snail pathway. Int J Oncol 46: 587-596, 2015. 